\title{
Corrected QT interval and QT dispersion in temporal lobe epilepsy
}

\author{
Maram Samy Nasef ${ }^{1}$, Ahmed Abdelmonem Gaber², Yousry Aboelnaga Abdelhamid², Islam Bastawy ${ }^{3}$,
} Salem Taha Abdelhady ${ }^{2}$ and Mona Mokhtar Wahid el $\operatorname{din}^{2^{*}}$ (D)

\begin{abstract}
Background: Cardiac arrhythmias are expected among patients with epilepsy due to the effect of anti-epileptic drugs. Temporal lobe epilepsy also causes autonomic seizures that may affect heart rhythm. Prolongation of the corrected QT interval and QT dispersion is a risk factor for cardiac arrhythmia.
\end{abstract}

Objectives: We aimed to assess corrected QT interval and QT dispersion in patients with epilepsy and if there is a difference between patients with temporal epilepsy versus non-temporal epilepsy.

Methods: This study was conducted on 100 patients (50 patients with temporal epilepsy and 50 patients with nontemporal epilepsy) and 50 age- and sex-matched healthy controls. They underwent a prolonged ( 6 to 24 h) 22 channel computerized electroencephalogram monitor with a 10-20 system. QT dispersion, QT interval, and corrected QT interval (using Bazett's formula) were calculated.

Results: This study showed significantly higher QT dispersion and corrected QT interval in patients with epilepsy when compared to the age- and sex-matched control group $(P<0.001, P<0.001)$. Also, the corrected QT interval and QT dispersion were significantly higher in temporal epilepsy patients when compared to the non-temporal group $(P<0.001, P<0.001)$.

Conclusion: Corrected QT interval and QT dispersion are higher in epileptic patients and more among temporal epilepsy patients in comparison to non-temporal epilepsy patients.

Keywords: Epilepsy, Temporal lobe, Corrected QT interval, QT dispersion, Arrhythmia, Sudden cardiac death

\section{Introduction}

Various studies found that people with epilepsy had a twofold to threefold increased risk of sudden cardiac arrest (SCA), irrespective of the traditional cardiac risk factors for SCA [1-3]. Ten to $50 \%$ of mortality in patients with chronic refractory epilepsy is sudden unexpected death in epilepsy (SUDEP). It most often affects young adults at the ages of 20-40 years. Despite the vigorous effort to study the pathophysiology of SUDEP today, it remains not fully understood [4]. It has been hypothesized that an intrinsic channelopathy may occur with sudden and intense

\footnotetext{
* Correspondence: Wahid_el_din@hotmail.com

${ }^{2}$ Neurology Department, Faculty of Medicine, Ain Shams University, Cairo, Egypt

Full list of author information is available at the end of the article
}

impulses directed from cortical areas, over-stimulating either the cardio-inhibitory center causing asystole or causing a massive adrenergic effect on the sympathetic pathways resulting in tachycardia [5].

Electrocardiogram (ECG) indicators for the risk of SCA include pathological cardiac repolarization such as shortening or prolongation of QT interval and increased QT dispersion (QTd) [6]. The QT interval is the measure of the duration of ventricular depolarization and repolarization [7]. Prolongation of ventricular repolarization can result in fatal ventricular arrhythmias [8]. Corrected QT interval (QTc) prolongation was found in inter-ictal ECGs of people with epilepsy more frequently than in those without epilepsy $[3,7,9]$ as well as in the post convulsive period of $2 \mathrm{~h}$ in some pediatric patients following 
generalized seizures [10]. Sadrnia and colleagues in 2010 proposed that there are some individuals more susceptible to develop a cardiac arrhythmia during seizures because of the effects of QT hysteresis and more sustained or increased prolongation of the QTc, particularly during prolonged unwitnessed nocturnal seizures with associated hypoxia [11].

Partial and generalized seizures often affect autonomic function during seizures as well as during the inter-ictal and post-ictal periods [12]. Sympathetic responses predominate during most seizures of either temporal or extra-temporal origin, causing tachycardia, tachypnea, increased blood pressure, pupillary dilatation, diaphoresis, and facial flushing [13]. Several studies suggest hemispheric lateralization of autonomic cardiovascular control. The right hemisphere may predominantly modulate sympathetic tone, whereas the left hemisphere modulates parasympathetic tone [14-16]. Ictal tachycardia may be more prominent after mesial temporal seizures [17, 18].

Our objective was to determine QTc and QTd through routine 12 lead ECG in epileptic patients and whether there is a difference in QTc or QTd between TLE and non-TLE.

\section{Methods}

This was an observational, cross-sectional 12-month study that took place in the period between October 2018 and October 2019. Our included patients were adults above 16 years of age with a diagnosis of epilepsy according to 2017 ILAE criteria [19]. Epileptic patients on medication known to cause prolonged QT interval (chlorpromazine; haloperidol; droperidol; quetiapine; olanzapine; amisulpride; thioridazine; types IA, IC, and III antiarrhythmics; tricyclic antidepressants; antihistamines; chloroquine; hydroxychloroquine; quinine; and macrolides) as well as epileptic patients who suffer hypokalemia, cardiac, hepatic, or renal diseases were excluded.

Patients were diagnosed as TLE based on seizure semiology, electroencephalogram (EEG), and magnetic resonance finding; all patients of the study underwent 12 lead ECG recording (CM 300 A, Comen, China); QT interval was measured from the onset of the QRS to the end of the $\mathrm{T}$ wave and was only measured in leads with a stable isoelectric line. If $\mathrm{U}$ waves were present, the QT interval was measured to the nadir of the curve between the $\mathrm{T}$ and U waves. QT interval was manually calculated [20].

QTc was calculated using Bazett's formula: QT/V RR. QTd was measured as the difference between the maximum and minimum QT interval [21]. A cardiologist analyzed all the ECGs.

The value of the QT interval was assessed according to the European Society of Cardiology guidelines which considers a QT interval to be prolonged when it is > $450 \mathrm{~ms}$ in males and $>470 \mathrm{~ms}$ in females [22]. Prolonged QTd was defined as prolonged if it is $>50 \mathrm{~ms}$ [20].

The collected data were coded, tabulated, and statistically analyzed using IBM SPSS statistics (Statistical Package for Social Sciences) software version 22.0, IBM Corp., Chicago, USA, 2013.

Descriptive statistics were done for quantitative data as minimum and maximum of the range as well as mean \pm standard deviation (SD) for quantitative normally distributed data, while it was done for qualitative data as number and percentage.

\section{Results}

We included 100 patients in our study: 50 patients diagnosed with temporal epilepsy; 50 with non-temporal epilepsy confirmed based on seizure semiology, EEG, and/ or radiological evidence; and 50 normal controls. Fiftyfour percent of our patients (54 patients) were males, and $46 \%$ (46 patients) were females. The age range was between 16 and 83 years with a mean age of $42.41 \pm$ 17.72 years. The patients had been suffering from the disease for a mean of 8.08 years (Table 1 ).

All patients underwent 12 lead ECG screening performed inter-ictal for comparison of the QT interval, QTc, and QTd between the study group and the control group. The mean QT interval in epilepsy patients was $411.06 \pm 39.23 \mathrm{~ms}$ while that of the control was $362.80 \pm$ $38.12 \mathrm{~ms}$, whereas the mean QTc of the control group $(411.60 \pm 33.20 \mathrm{~ms})$ was also much lower than that of the epilepsy group $473.28 \pm 39.56 \mathrm{~ms}$. And the calculated QTd was much higher in the epilepsy group with a mean of $45.16 \pm 21.52 \mathrm{~ms}$ while that of control was $22.40 \pm 10.21 \mathrm{~ms}$. Meaning that the QT interval, QTc, and QTd values were significantly higher in the epilepsy group $(P$ value $<0.001)$ than that of age- and sexmatched control (Fig. 1).

There was no statistically significant effect for gender on either of QTc or QTd values. There was a statistically significant effect of duration of illness on QT interval

Table 1 Basic descriptive data of the study group

\begin{tabular}{|c|c|c|c|c|}
\hline & $\begin{array}{l}\text { Control } \\
n=50\end{array}$ & $\begin{array}{l}\text { Cases } \\
n=100\end{array}$ & $\begin{array}{l}\text { Test } \\
\text { value }\end{array}$ & $\begin{array}{l}P \\
\text { value }\end{array}$ \\
\hline \multicolumn{5}{|l|}{ Age } \\
\hline Mean \pm SD & $42.22 \pm 19.0$ & $42.50 \pm 17.0$ & -0.091 & 0.928 \\
\hline Range & $16-83$ & $16-83$ & & \\
\hline \multicolumn{5}{|l|}{ Sex } \\
\hline Male & 24 (48.0\%) & $46(46.0 \%)$ & 0.054 & 0.817 \\
\hline Female & $26(52.0 \%)$ & $54(54.0 \%)$ & & \\
\hline \multicolumn{5}{|c|}{ Duration of illness } \\
\hline Mean \pm SD & 0.0 & $8.08 \pm 9.14$ & 0.0 & 0.0 \\
\hline
\end{tabular}




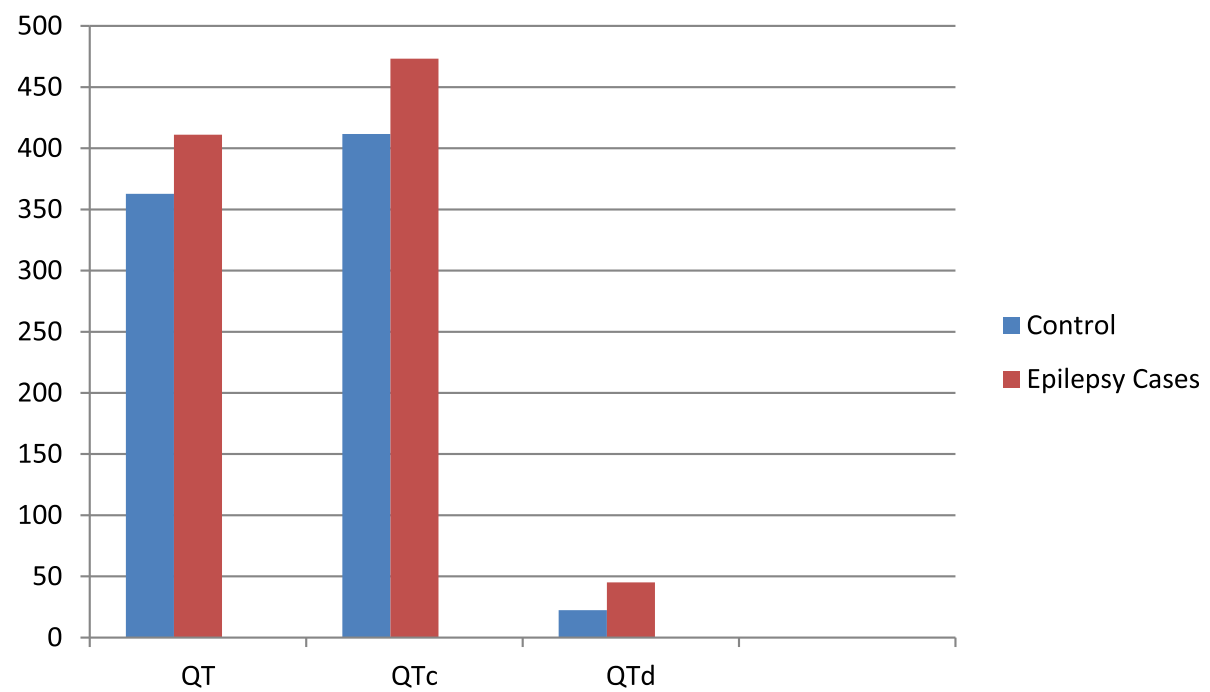

Fig. 1 Comparison between control and epilepsy cases as regards QT, QTc, and QTd

and QTc. The greater the duration of illness, the longer the QT interval ( $P$ value 0.024$)$ and QTc ( $P$ value 0.017 ), while there was no significant effect on QTd due to the duration of illness.

TLE group had a significantly higher value of QT interval, QTc, and QTd than that of control. It had higher QT interval values than control with a mean of $414.20 \pm 39.18 \mathrm{~ms}$ while that of control was $362.80 \pm$ $38.12 \mathrm{~ms}$. QTc was also higher in the TLE group as its mean was $482.08 \pm 42.86 \mathrm{~ms}$ while that of control was $411.60 \pm 33.20 \mathrm{~ms}$. The calculated QTd was also significantly higher in the TLE group as its mean was $49.60 \pm$ $21.19 \mathrm{~ms}$ while that of control was $22.40 \pm 10.21 \mathrm{~ms}$ (Table 2).

On comparing non-TLE with controls, we found statistically significantly higher QT interval, QTc, and QTd values in the non-temporal epilepsy group when compared to those of the control. The mean QT interval of the non-TLE was $407.92 \pm 39.41 \mathrm{~ms}$ while that of the control was $362.80 \pm 38.12 \mathrm{~ms}$. The QTc was also higher in the non-TLE as it showed a mean of $464.48 \pm 34.17$ ms whereas that of the control was $411.60 \pm 33.20 \mathrm{~ms}$. The QTd of the control was much lower than the nontemporal group with a mean of $22.40 \pm 10.21 \mathrm{~ms}$ while that of the non-temporal was $40.72 \pm 21.13 \mathrm{~ms}$ (Table 2). Additionally, on comparing QTc and QTd between TLE and non-TLE groups, these values were significantly higher in the TLE group of patients $(P=0.025$ and $P=$ 0.038).

In our study, $72 \%$ of our patients had focal seizures (50 patients with temporal lobe epilepsy and 22 patients with extra-temporal lobe epilepsy), and $28 \%$ had

Table 2 Comparison between temporal, non-TLE, and control as regards QT, QTC, and QTd

\begin{tabular}{|c|c|c|c|c|}
\hline & $\begin{array}{l}\text { Control group } \\
n=50\end{array}$ & $\begin{array}{l}\text { TLE } \\
n=50\end{array}$ & $\begin{array}{l}\text { Non TLE } \\
n=50\end{array}$ & $\begin{array}{l}P \\
\text { value }\end{array}$ \\
\hline \multicolumn{5}{|l|}{$\overline{Q T}$} \\
\hline Mean \pm SD & $362.80 \pm 38.12$ & $414.20 \pm 39.18$ & $407.92 \pm 39.41$ & $<0.001$ \\
\hline Range & $240-440$ & $320-480$ & $320-520$ & \\
\hline \multicolumn{5}{|l|}{ QTC } \\
\hline Mean \pm SD & $411.60 \pm 33.20$ & $482.08 \pm 42.86$ & $464.48 \pm 34.17$ & $<0.001$ \\
\hline Range & $309-487$ & $397-596$ & $413-571$ & \\
\hline \multicolumn{5}{|l|}{ QTd } \\
\hline Mean \pm SD & $22.40 \pm 10.21$ & $49.60 \pm 21.19$ & $40.72 \pm 21.13$ & $<0.001$ \\
\hline Range & $10-40$ & $10-100$ & $0-100$ & \\
\hline
\end{tabular}


generalized seizures; only 8 patients showed evident ictal or post-ictal autonomic manifestations mainly in the form of tachyarrhythmia and sweating. The mean QT and QTc values for focal epilepsy were 412.9 and 476.7 ms respectively, while the mean QT and QTc values for generalized epilepsy were 412 and $472 \mathrm{~ms}$. Patients with evident ictal or post-ictal autonomic manifestations had a mean QT value of $390 \mathrm{~ms}$ and a QTc of $449 \mathrm{~ms}$. We found no statistically significant difference in the QT and QTc values between patients having generalized, focal, or other seizure types.

Ten patients had MRI pathology, 8 patients showed evidence of temporal sclerosis, one patient with cortical dysplasia in the right temporal lobe, and one patient with low-grade glioma in the right high parietal region.

\section{Discussion}

We aimed in our study to evaluate cardiac repolarization indices in epileptic patients inter-ictal especially those with TLE patients in comparison to non-TLE patients through routine 12 lead surface ECG as epileptic seizures increase the risk of cardiac arrhythmias aiming for a better understanding of the pathophysiology of SUDEP. Although Holter monitoring could accurately measure QTc and QTd with added data on temporal variation in QTc and QTd rather than only spatial variation assessed by ECG [23], in practice, it is easier to evaluate cardiac repolarization indices using 12 lead surface ECG.

In our study, QT interval values in epileptic patients were higher than that of the control, with prolonged QT interval found in $10 \%$ of cases denoting increased risk of ventricular arrhythmia especially in the presence of cardiac structural changes as ischemic heart disease, heart failure, ventricular hypertrophy, or autonomic nervous system dysfunction eventually leading to sudden death. Also, we found that the epilepsy group had higher QTc values and prolongation of the QTd values than that of age- and gender-matched controls with $51 \%$ of our epileptic patients (27 males and 24 females) having QTc prolongation. Previous studies showed prolonged repolarization indices in epileptic patients in comparison to healthy controls as Asoğlu and his colleagues in 2020 who studied generalized tonic-clonic seizure patients during the inter-ictal period, and they found prolonged QTd in epileptic patients when compared to the healthy group [24]. Also, Dagar and his colleagues in 2020 found a prolonged QTc and QTd in epileptic patients in the inter-ictal period in comparison to healthy controls [25]. Moreover, Brotherstone and his colleagues in 2010 found that QTc increased significantly in epileptic patients during seizures in different forms of epilepsy [9]. These changes in ventricular repolarization may be explained by animal studies that showed altered cardiac expression of some sodium, potassium, calcium, and cationic channels resulting in acquired channelopathies that may increase arrhythmic risk in epileptic patients and eventually increased sudden death risk [26]. What supports this explanation is a postmortem study that studied SUDEP and concluded that the most likely pathogenic genetic abnormality on molecular autopsy was channelopathies [27]. Also, this overlap between prolongation of QT interval and epilepsy was further discussed in several studies that mentioned that there is an association between some congenital long QT syndromes and epilepsy [28].

Moreover, we found that patients with epileptogenic discharge in EEG showed significant prolongation in the QTc values with no effect on QTd values. A single study conducted on 11 patients who developed later SUDEP showed prolonged QTc during ictal discharge than without ictal discharge [29]. Although it is expected to find that seizure frequency and control should affect QT interval, there was conflicting data around this point. Several studies found that it did not affect QT interval values [3,30], yet Asadollahi and colleagues, in 2019, found a shorter QTc and a longer QTd on his study performed on patients with refractory epilepsy patients, while other studies found that there was significant QTc prolongation in patients with uncontrolled seizures [22]. However, previous reviews stated that cardiac electrophysiological changes in epileptic patients are more related to long-standing and uncontrolled epilepsy which is consistent with our finding of significantly prolonged QTc with a longer duration of illness [31].

There is a difference in the normal values of QTc between males and females and this may be attributed to the effect of male sex hormone effect on shortening of action potential duration and QT interval, and this is associated with generally increased risk of prolonged QTdependent arrhythmia in females as this risk depends on the prolongation of repolarization indices [32]. Despite this variation, in our study, there was no gender effect on QTc or QTd prolongation in epileptic patients in comparison to the control group.

Patients with TLE were found to have a significant prolongation of QTc and QTd in comparison to nonTLE patients, and this may be explained by Lathers and colleagues, in 1987, as they described a mechanism called the lockstep phenomenon, based on a study of an anesthetized cat with alpha-chloralose, received pentylenetetrazol (a drug that cause convulsions in high doses), the cardiac postganglionic sympathetic and vagal nerve discharges were correlated with the inter-ictal spikes. The changes during an epileptiform discharge were shown to alter the sympathetic activity and caused an alteration of peripheral efferent discharge to the heart [30]. This may be explained by the more autonomic 
dysregulation associated with TLE among epileptic patients [33]. Features of autonomic dysregulation seen in association with TLE include sympathetic and parasympathetic overactivation increasing the risk of SUDEP through disturbed neuro-respiratory and neuro-cardiac connections [34]. The suggested pathophysiological mechanism of QT interval prolongation with increased sympathetic activity relates to early after depolarization [35]. Sympathetic activity increases with uncontrolled disease due to parasympathetic dysfunction that improves with disease control, and this may explain the more QTc prolongation with the chronic uncontrolled disease [36]. Moreover, increased sympathetic activity with TLE is also associated with increased myocardial stiffness, increased atrial volumes, and ventricular pressures denoting increasing risk of structural heart disease in addition to the arrhythmic risk that together may be considered the most accepted explanations for SUDEP [37].

\section{Limitations}

Temporal lobe epilepsy was diagnosed depending on semiology and inter-ictal EEG only; however, autonomic seizures may arise from activation of extratemporal areas.

\section{Conclusion}

QTc interval and QTd are higher in epileptic patients more among TLE patients in comparison to non-TLE.

\section{Recommendations}

Follow-up ECG is recommended in epileptic patients and use of QT interval diary, particularly TLE.

\section{Abbreviations}

ECG: Electrocardiogram; EEG: Electroencephalogram; QTc: Corrected QT interval; QTd: QT dispersion; SCA: Sudden cardiac arrest; SD: Standard deviation; SUDEP: Sudden unexpected death in epilepsy; TLE: Temporal lobe epilepsy

\section{Acknowledgements}

None

\section{Authors' contributions}

The authors read and approved the final manuscript. MSN: recruitment and data collection. AAG and YAA: statistical design and manuscript review. IB: interpreting ECG findings and manuscript writing. STA: manuscript revision, data collection, and statistical analysis. MMW: study design, data collection, and manuscript writing.

\section{Funding}

No funds were received to fulfil this work.

\section{Availability of data and materials}

All data and materials that support findings in this manuscript are available. Please contact the author for data requests.

\section{Ethics approval and consent to participate}

The procedures followed were in accordance with the ethical standards of the responsible committee on human experimentation and with the principles of Helsinki Declaration. Informed written consent was obtained from all participants, and ethical committee permission from the Neuropsychiatry Department of Ain Shams University on January 9, 2018.

Consent for publication

Not applicable

\section{Competing interests}

The authors declare that they have no competing interests.

\section{Author details}

${ }^{1}$ Faculty of Medicine, Cairo University, Cairo, Egypt. ${ }^{2}$ Neurology Department, Faculty of Medicine, Ain Shams University, Cairo, Egypt. ${ }^{3}$ Cardiology

Department, Faculty of Medicine, Ain Shams University, Cairo, Egypt.

Received: 16 July 2020 Accepted: 8 December 2020

Published online: 07 January 2021

\section{References}

1. Shankar R, Cox D, Jalihal V, Brown S, Hanna J, McLean B. Sudden death in epilepsy (SUDEP): development of a safety checklist. Seizure. 2013;22:812-7.

2. Wilder-Smith E, Lim SH. Heart rate changes during partial seizures: a study amongst Singaporean patients. BMC Neurol. 2001;1:5..

3. Lamberts R, Blom M, Novy J, Belluzzo M, Seldenrijk A, Penninx B, et al. Increased prevalence of ECG markers for sudden cardiac arrest in refractory epilepsy. J Neurol Neurosurg Psychiatry. 2015;86:309-13..

4. Thom M. Neuropathology of epilepsy: epilepsy-related deaths and SUDEP. Diagn Histopathol. 2019;25(1):23-33..

5. Ansakorpi H, Korpelainen J, Tanskanen P, Huikuri H, Koivula A, Tolonen U, et al. Cardiovascular regulation and hippocampal sclerosis. Epilepsia. 2004; 45:933-9.

6. Shmuely S, Van der Lende S, Lamberts R, Sander J, Thijs R. The heart of epilepsy: current views and future concepts. Seizure. 2017;44:176-83..

7. Surges R, Taggart P, Sander J, Walker M. Too long or too short? New insights into abnormal cardiac repolarization in people with chronic epilepsy and its potential role in sudden unexpected death cardiac repolarization: basic mechanism and clinical. Epilepsia. 2010;5:738-44..

8. Mohebi R, Jehan A, Grober A, Froelicher V. Percentile categorization of QT interval as an approach for identifying adult patients at risk for cardiovascular death. Heart Rhythm. 2017;8:1210-6..

9. Brotherstone R, Blackhall B, McLellan A. Lengthening of corrected QT during epileptic seizures. Epilepsia. 2010;51(2):221-32..

10. Kandler L, Fieldler A, Scheer K, Wild F, Frick U, Schneider P. Early postconvulsive prolongation of QT time in children. Acta Paediatr. 2005;94:1243$7 .$.

11. Sadrnia S, Yousefi P, Jalali L. Correlation between seizure in children and prolonged QT interval. ARYA Atheroscler. 2013:9:7-10.

12. Devinsky O. Effects of seizures on autonomic and cardiovascular function. Epilepsy Curr. 2004;4(2):43-6.

13. Van Buren J. Some autonomic concomitants of ictal automatism. Brain. 1958:81:505-29..

14. Wittling W, Block A, Genzel S, Schweiger E. Hemisphere asymmetry in parasympathetic control of the heart. Neuropsychologia. 1998:36:461-8.

15. Oppenheimer S, Gelb A, Girvin J, Hachinski V. Cardiovascular effects of human insular cortex stimulation. Neurology. 1992;42:1727-32..

16. Hilz M, Dutsch M, Perrine K, Rauhut U, Devinsky O. Hemispheric influence on autonomic modulation and baroreflex sensitivity. Ann Neurol. 2001;49: 575-84..

17. Leutmezer F, Schernthaner C, Lurger S, Potzelberger K, Baumgartner $C$. Electrocardiographic changes at the onset of epileptic seizures. Epilepsia. 2003:44:348-54..

18. Nei M, Ho R, Sperling M. EKG abnormalities during partial seizures in refractory epilepsy. Epilepsia. 2000;41:542-8..

19. Scheffer I, Berkovic S, Capovilla G, Connolly M, French J, Guilhoto L, et al. ILAE classification of the epilepsies: position paper of the ILAE Commission for Classification and Terminology. Epilepsia. 2017;58(4):512-21..

20. Christensen P, Gall M, Major-Pedersen A, Sato A, Rossing P, Breum L, et al. QTc interval length and QT dispersion as predictors of mortality in patients with non-insulin-dependent diabetes. Scand J Clin Lab Invest. 2000;60(4): 323-32.. 
21. Li H, Ho L, Wang D, Shu J, Cui C, Han F, et al. High voltage J-waves as a predictor of death in acute ST-segment elevated myocardial infarction in hospital. Cardiol Plus. 2019;4(1):10-4..

22. Asadollahi M, Shahidi M, Ramezani M, Mehdi SM. Inter-ictal electrocardiographic alternations in patients with drug-resistant epilepsy. Seizure. 2019;69:7-10..

23. DE Maria E, Curnis A, Garyfallidis P, Mascioli G, Santangelo L, Calabrò R, et al. QT dispersion on ECG Holter monitoring and risk of ventricular arrhythmias in patients with dilated cardiomyopathy. Heart Int. 2006;2(1):33..

24. Asoğlu R, Özdemir M, Aladağ N, Asoğlu E. Evaluation of cardiac repolarization indices in epilepsy patients treated with carbamazepine and valproic acid. Medicina (Kaunas). 2020;56(1):20. https://doi.org/10.3390/ medicina56010020 PMID: 31935975; PMCID: PMC7022319..

25. Dagar S, Emektar E, Corbacioglu SK, Demirci OL, Tandogan M, Cevik Y. Evaluation of electrocardiographic parameters in patients with epileptic seizure. Acta Neurol Belg. 2020;120(2):321-7. https://doi.org/10.1007/s1376011019-01182-8 Epub 2019 Jul 11. PMID: 31297670.

26. Li MCH, O'Brien TJ, Todaro M, Powell KL. Acquired cardiac channelopathies in epilepsy: evidence, mechanisms, and clinical significance. Epilepsia. 2019; 60(9):1753-67.

27. Chahal CAA, Salloum MN, Alahdab F, Gottwald JA, Tester DJ, Anwer LA, et al. Systematic review of the genetics of sudden unexpected death in epilepsy: potential overlap with sudden cardiac death and arrhythmiarelated genes. J Am Heart Assoc. 2020;9(1):e012264..

28. Manolis TA, Manolis AA, Melita H, Manolis AS. Sudden unexpected death in epilepsy: the neuro-cardio-respiratory connection. Seizure. 2019;64:65-73.

29. de Sousa J, Fialho G, Wolf P, Walz R, Lin K. Determining factors of electrocardiographic abnormalities in patients with epilepsy: a case-control study. Epilepsy Res. 2017;129:106-16..

30. Lathers C, Schraeder P, Weiner F. Synchronization of cardiac autonomic neural discharge with epileptogenic activity: the lock step phenomenon Electroencephalogr Clin Neurophysiol. 1987;67:247-59.

31. Ravindran K, Powell KL, Todaro M, O'Brien TJ. The pathophysiology of cardiac dysfunction in epilepsy. Epilepsy Res. 2016;127:19-29.

32. Salama G, Bett GC. Sex differences in the mechanisms underlying long QT syndrome. Am J Physiol Heart Circ Physiol. 2014;307(5):H640-8..

33. Freeman R, Schachter SC. Autonomic epilepsy. Semin Neurol. 1995;15(2): 158-66.

34. TA MAA, Melita H, Manolis AS. Sudden unexpected death in epilepsy: the neuro-cardio-respiratory connection. Seizure. 2019;64:65-73.

35. Huffaker R, Lamp ST, Weiss JN, Kogan B. Intracellular calcium cycling, early after depolarizations, and reentry in simulated long QT syndrome. Heart Rhythm. 2004;1(4):441-8.

36. Toichi M, Murai T, Sengoku A, Miyoshi K. Inter-ictal change in cardiac autonomic function associated with EEG abnormalities and clinical symptoms: a longitudinal study following acute deterioration in two patients with temporal lobe epilepsy. Psychiatry Clin Neurosci. 1998;52(5): 499-505..

37. Fialho GL, Wolf $P$, Walz R, Lin K. Increased cardiac stiffness is associated with autonomic dysfunction in patients with temporal lobe epilepsy. Epilepsia. 2018;59(6):e85-90

\section{Publisher's Note}

Springer Nature remains neutral with regard to jurisdictional claims in published maps and institutional affiliations.

\section{Submit your manuscript to a SpringerOpen ${ }^{\circ}$ journal and benefit from:}

- Convenient online submission

- Rigorous peer review

- Open access: articles freely available online

- High visibility within the field

- Retaining the copyright to your article

Submit your next manuscript at $\boldsymbol{\nabla}$ springeropen.com 